San Jose State University

SJSU ScholarWorks

Faculty Publications

Physics and Astronomy

September 2014

\title{
Magnetic Correlations and Pairing in the 1/5-Depleted Square Lattice Hubbard Model
}

\author{
Ehsan Khatami \\ San Jose State University, ehsan.khatami@sjsu.edu \\ Rajiv R.P. Singh \\ University of California, Davis \\ Warren E. Pickett \\ University of California, Davis \\ Richard T. Scalettar \\ University of California, Davis
}

Follow this and additional works at: https://scholarworks.sjsu.edu/physics_astron_pub

Part of the Atomic, Molecular and Optical Physics Commons

\section{Recommended Citation}

Ehsan Khatami, Rajiv R.P. Singh, Warren E. Pickett, and Richard T. Scalettar. "Magnetic Correlations and Pairing in the 1/5-Depleted Square Lattice Hubbard Model" Physical Review Letters (2014): 106402. https://doi.org/10.1103/PhysRevLett.113.106402

This Article is brought to you for free and open access by the Physics and Astronomy at SJSU ScholarWorks. It has been accepted for inclusion in Faculty Publications by an authorized administrator of SJSU ScholarWorks. For more information, please contact scholarworks@sjsu.edu. 


\title{
Magnetic Correlations and Pairing in the 1/5-Depleted Square Lattice Hubbard Model
}

\author{
Ehsan Khatami, ${ }^{1,2}$ Rajiv R. P. Singh, ${ }^{1}$ Warren E. Pickett, ${ }^{1}$ and Richard T. Scalettar \\ ${ }^{1}$ Department of Physics, University of California, Davis, California 95616, USA \\ ${ }^{2}$ Department of Physics and Astronomy, San Jose State University, San Jose, California 95192, USA
}

(Received 15 April 2014; published 4 September 2014)

\begin{abstract}
We study the single-orbital Hubbard model on the 1/5-depleted square-lattice geometry, which arises in such diverse systems as the spin-gap magnetic insulator $\mathrm{CaV}_{4} \mathrm{O}_{9}$ and ordered-vacancy iron selenides, presenting new issues regarding the origin of both magnetic ordering and superconductivity in these materials. We find a rich phase diagram that includes a plaquette singlet phase, a dimer singlet phase, a Néel and a block-spin antiferromagnetic phase, and stripe phases. Quantum Monte Carlo simulations show that the dominant pairing correlations at half filling change character from $d$ wave in the plaquette phase to extended $s$ wave upon transition to the Néel phase. These findings have intriguing connections to ironbased superconductors, and suggest that some physics of multiorbital systems can be captured by a singleorbital model at different dopings.
\end{abstract}

DOI: 10.1103/PhysRevLett.113.106402

The interplay of magnetic order and pairing correlations has been a central topic in strongly correlated materials, and, in particular, in copper-based [1] and more recently iron-based [2] high-temperature superconductors. That pairing arises in intimate proximity to magnetism is initially somewhat surprising, since long-range magnetic order usually leads to an insulating Mott or Slater gap, which precludes superconductivity. Much study of these materials has been devoted to understanding how doping, and the presence of multiple bands, modify the magnetism [3-5] and allow pairing and short-range spin order to complement each other [6-16].

One geometry which has been a recurring structure in real materials, and which permits tuning of the degree of magnetic order, is the periodically $1 / 5$-depleted square lattice, consisting of coupled plaquette unit cells (see Fig. 1) [17-21]. It was first discovered in the study of spin-gap calcium vanadate material $\mathrm{CaV}_{4} \mathrm{O}_{9}$ [22]. More recently, the same structure arises in an ordered vacancy iron selenide family of materials [23,24] where metallic, insulating, multiple magnetically ordered, and superconducting phases arise [14-16,25-27].

An itinerant Hubbard model in this geometry with a single orbital per site is a four-band model and can be mapped onto a four plaquette-orbitals model on the nondepleted square lattice. Such a model allows a systematic exploration of (i) crossovers from weak to strong coupling behavior, (ii) multiple competing magnetic and spin-gap phases, (iii) possible effects of proximity to different phase transitions on the superconducting pairing, and (iv) connections between a model with multiple orbitals per site and a single-orbital model at different dopings. These make it an important model conceptually, and very relevant to the iron selenide family of materials.

Here, we study this single-orbital Hubbard Hamiltonian on the $1 / 5$-depleted square lattice. Using the determinant
PACS numbers: 71.10.Fd, 74.20.Rp, 74.70.Xa, 75.40.Mg

quantum Monte Carlo (DQMC) method [28,29], which has no "minus-sign problem" [33] at half filling on this lattice, we find that the dimerized phase of the large Hubbard $U$ limit (Heisenberg model) connects smoothly to a band insulator as $U$ goes to zero. However, as $U$ decreases, the Néel phase extends farther and farther into the region where intraplaquette hopping is dominant. The plaquette phase, at large $U$, is always separated from the metallic phase, obtained at $U=0$, by an intervening Néel phase.

In the limit where the interplaquette hopping $t^{\prime}$ is much smaller than the intraplaquette hopping $t$, our model is a variant of the weakly coupled plaquette model studied by Tsai and Kivelson [34]. This Hamiltonian can be rigorously shown to have pair binding and a superconducting phase at infinitesimal doping away from half filling for $U \lesssim 4.6 t$, a property which remains true for our model as well. Our QMC simulations extend the result away from small $t^{\prime}$ and demonstrate that singlet pairing is predominantly in the

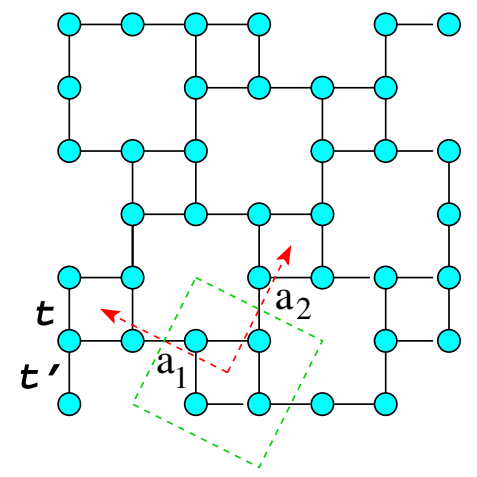

FIG. 1 (color online). The geometry of the $1 / 5$-depleted square lattice. $2 \times 2$ plaquettes have intersite hopping $t$. Different plaquettes are linked by hopping $t^{\prime}$. The two primitive vectors are shown by red arrows and the unit cell is shown by the tilted square. 
$d$-wave channel in the plaquette phase and becomes particularly large as $U$ exceeds one half of the noninteracting bandwidth. We also find that, surprisingly, as soon as one approaches the phase transition to the Néel order, the dominant pairing changes from $d$ wave to extended $s$ wave. To our knowledge, there has been no previous unbiased demonstration of interchange of superconducting pairing symmetry with change in the magnetic properties, emphasizing the close interplay of magnetic and superconducting correlations in these systems.

Indeed, this observation has possible connections to the iron selenide materials whose magnetic phases include ubiquitous stripe phases and a $2 \times 2$ block-spin antiferromagnet $[23-25,35,36]$. In the latter, spins within a plaquette align, and these block spins then order in an antiferromagnetic pattern. Using the random phase approximation (RPA), we have explored a number of different magnetic instabilities in the 1/5-depleted geometry. At half filling, the dominant order in our nearest-neighbor (NN) model is the usual Néel phase. However, away from half filling, both the stripe phase and the $2 \times 2$ block-spin antiferromagnet are found to be the leading instabilities over different doping ranges, remarkably, showing that such phases can arise in models without any frustration or multiorbital character.

The Hubbard Hamiltonian considered here is

$$
\begin{aligned}
\hat{H}= & -\sum_{i j \sigma} t_{i j} c_{i \sigma}^{\dagger} c_{j \sigma}-\mu \sum_{i \sigma} n_{i \sigma} \\
& +U \sum_{i}\left(n_{i \uparrow}-\frac{1}{2}\right)\left(n_{i \downarrow}-\frac{1}{2}\right) .
\end{aligned}
$$

Here, $c_{i \sigma}\left(c_{i \sigma}^{\dagger}\right)$ annihilates (creates) a fermion with spin $\sigma$ on site $i, n_{i \sigma}=c_{i \sigma}^{\dagger} c_{i \sigma}$ is the number operator, $U$ is the on-site repulsive Coulomb interaction, and $t_{i j}$ is the hopping matrix element between sites $i$ and $j$. We allow for NN hopping only and consider two different values: $t_{i j}=t$ if $i$ and $j$ are nearest neighbors within a plaquette, and $t_{i j}=t^{\prime}$ if $i$ and $j$ are nearest neighbors on a bond that connects two distinct plaquettes. At $U=0$, there are four bands with dispersion $\epsilon_{\alpha}(k)$ given by the roots of $\left[\epsilon_{\alpha}^{2}(k)-t^{\prime 2}\right]^{2}-4 t^{2}\left[\epsilon_{\alpha}(k)+t^{\prime} \cos k_{x}\right]\left[\epsilon_{\alpha}(k)+t^{\prime} \cos k_{y}\right]=0$. As we vary the ratio $t^{\prime} / t$, the noninteracting bandwidth $w=$ $4 t+2 t^{\prime}$ is kept fixed at 6 , setting the energy unit to $w / 6$ throughout the paper.

The richness of the band structure has prompted a recent mean-field study of the model at quarter filling, where there is on average one half particle per site [37]. When $t^{\prime}=t$, the Fermi energy at this filling coincides with a Dirac cone structure at the zone center and a flat band in its proximity. Yasufumi et al. [37] identify three different phases: a paramagnetic insulator, a paramagnetic metal, and an antiferromagnet, for which phase transitions could be described by an effective SU(3) theory. The Mott transition in the dimer region has also been recently studied within a cluster dynamical mean-field theory [38].
The phase diagram at half filling in the plane of $t^{\prime} / t$ and $U /(1+U)$ is given in Fig. 2. It establishes the dominant magnetic instability as antiferromagnetism. The range of $t^{\prime} / t$ for which the ground state is Néel ordered is shown as thick horizontal lines for three different values of $U$. At $U \ll 1$, the antiferromagnetic (AF) region extends from an infinitesimal $t^{\prime}$ all the way to $t^{\prime} / t=2$, beyond which the noninteracting system is a band insulator. The Néel phase in this regime is favored by AF nesting at the Fermi surface for $t^{\prime}<2 t$, and the fact that the growing nested area compensates for the loss of uniformity in the system as $t^{\prime} / t \rightarrow 0$. We obtain this range from the RPA, which is exact in that limit (the RPA estimate for the AF phase boundary at nonzero $U$ is also shown by a dashed line in Fig. 2).

As we turn on the interaction, we find that for $t$ and $t^{\prime}$ sufficiently close to each other, there is always a nonzero Néel order parameter in the thermodynamic limit. We locate the phase boundary by finite-size scaling of the DQMC AF structure factor, $S_{\mathrm{AF}}$ [29]. One can see that as $U$ increases, the Néel ordered region shrinks, especially on the plaquette side, and moves to the Heisenberg limit $(U \rightarrow \infty)$ range [19].

Also shown as filled circles in the phase diagram of Fig. 2 are the hopping ratios at which the intra- and interplaquette NN spin correlations are equal in magnitude. This line of

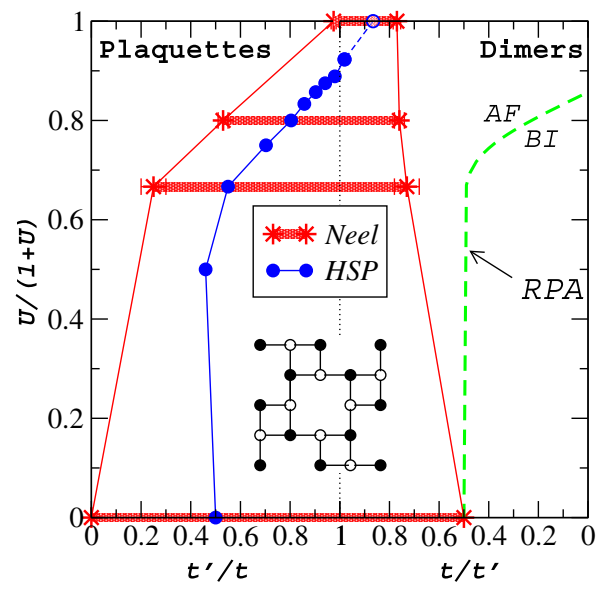

FIG. 2 (color online). Ground state phase diagram at half filling. The thick horizontal lines indicate the region with long-range Néel order. At $U=\infty$, the AF region is obtained from Ref. [19] (Heisenberg model study on the same geometry) by considering $t^{\prime} / t=\sqrt{J^{\prime} / J}\left(J\right.$ and $J^{\prime}$ are the intra- and interplaquette spin exchange interactions). Blue circles track the high symmetry point (HSP) inside the AF region where the NN spin correlations are equal on all bonds [see Fig. 3(a)]. Similar results for the HSP are not available for the Heisenberg limit. So instead, the empty blue circle indicates the location of the maximum of the AF moment in that limit. The dashed line shows the AF-band insulator (BI) phase boundary as predicted by the RPA. The inset shows the AF ordering. Filled (empty) circles denote up (down) spins. 
"high symmetry points" (HSPs) favors the plaquette side of the phase diagram until it veers toward the dimer side around $U=3$, tracking the magnetically ordered region.

Figure 3(a) shows the absolute value of the difference of NN spin correlations on the two types of bonds at inverse temperature $\beta=20$ as a function of hopping ratios. At the weakest coupling $U=1$ the $\mathrm{NN}$ spin correlation on the intraplaquette $t$ bonds exceeds the interplaquette $t^{\prime}$ bonds up to $t^{\prime} / t \sim 0.5$, at which point the relative size is reversed [39]. However, at the strongest coupling studied, $U=12$, the intraplaquette spin correlation remains larger all the way to $t^{\prime} / t \sim 1$. The finite-size dependence of these correlations is either negligible, or has been taken into account [29] (see caption of Fig. 3 for details). We note that all of the calculated NN spin correlations are antiferromagnetic, regardless of the value of $t^{\prime} / t$ or $U$.

The results in Fig. 3(b) show the low-temperature $S_{\mathrm{AF}}$ as a function of the hopping ratios for the same range of
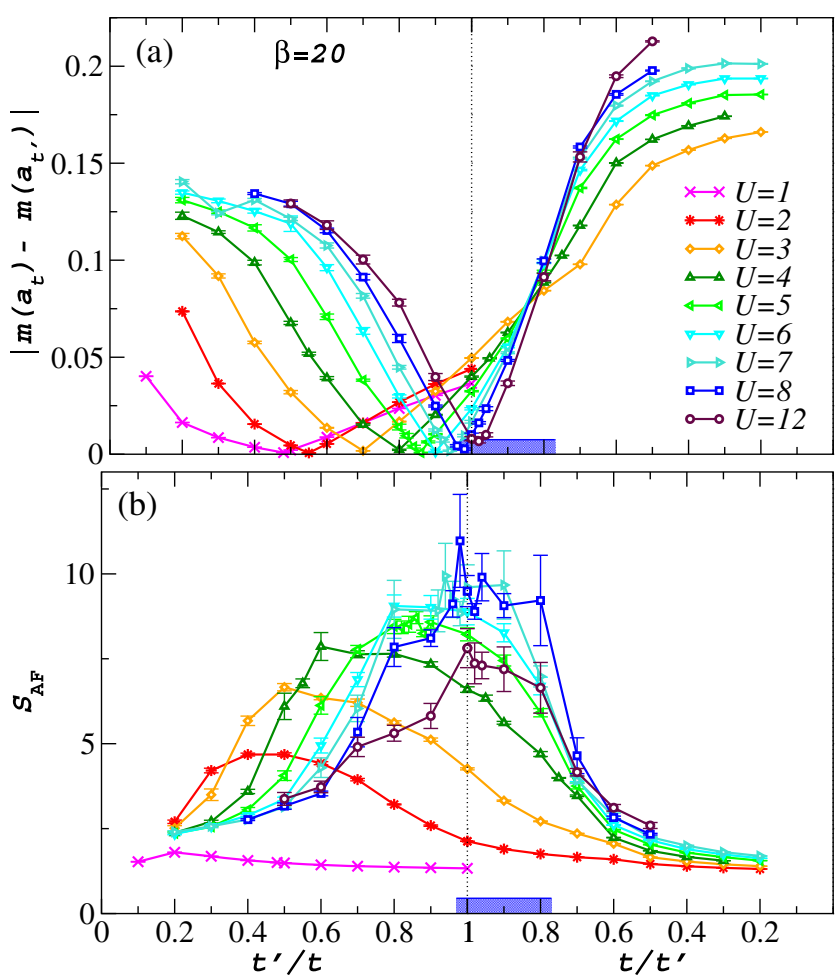

FIG. 3 (color online). (a) The absolute value of the difference in the NN spin correlations on $t$ and $t^{\prime}$ bonds at $\beta=20$ from DQMC vs $t^{\prime} / t$ for several values of the interaction strength $(m(\mathbf{r})$ is the spin-spin correlation function at distance $\mathbf{r}$ and "a" denotes the lattice constant between NNs [29]). The shaded region on the horizontal axis is added to show the boundaries of the Néel phase in the $U \rightarrow \infty$ limit. The lattice is a $4 \times 4$ arrangement of $2 \times 2$ plaquettes $(N=64)$, except for $U=1$ and 2 where the $8 \times 8$ arrangement $(N=256)$ is used. We have also simulated a 576-site lattice for the latter interactions and found no significant changes in the location of the HSP. (b) The AF structure factor vs $t^{\prime} / t$ at $\beta=20$ from DQMC. Except for $U=1$, for which $N=256$, the results are obtained for the $N=64$ lattice. interaction strengths as in Fig. 3(a). Although these results are for a single (relatively large) lattice size, the evolution of the peak of $S_{\mathrm{AF}}$ clearly conveys the trend in the long-range order as $U$ is increased towards the Heisenberg limit. These maxima shift steadily from the plaquette side at weak coupling to the dimer side at strong coupling.

An intriguing feature seen in this model is the change in symmetry of low-temperature pairing correlations from $d$ wave in the plaquette phase to extended $s$ wave upon entering the Néel phase. This is demonstrated in Fig. 4, where we plot the uniform pairing structure factor [29] for the two symmetries versus the interaction strength at $t^{\prime} / t=0.3$, and vs the hopping ratio at $U=4$, for which we know the location of the AF phase transitions. As shown in Fig. 4, finite-size effects at small $U$ are not responsible for this difference. We have also verified that the values of the structure factor do not change significantly by further lowering the temperature. At $U=4$, the change in the pairing symmetry takes place inside the AF region just before the transition to the plaquette phase. For all the other interaction strengths, the location of this crossover appears to fall to the right (larger $t^{\prime}$ side) of the AF phase boundary. As the charge gap is nonzero in both the $\mathrm{AF}$ and the plaquette phase, we do not expect to find superconductivity at half filling. However, the strength of the pairing at half filling should be indicative of the nature of superconductivity upon doping. The $d$-wave pairing in the weakly coupled plaquette phase agrees with the general arguments of Scalapino and Trugman [40] and of Tsai and Kivelson [34]. The dominance of extended $s$-wave pairing near the phase transition is a surprising result and points to the close interplay between magnetism and superconductivity in these systems.

We now turn to the case away from half filling, where we use the RPA instead of the DQMC method, as lowtemperature results are not available for the latter. The RPA, which is reasonably accurate only at weak couplings, can offer insight into the competition between different magnetic orderings that this geometry may favor in

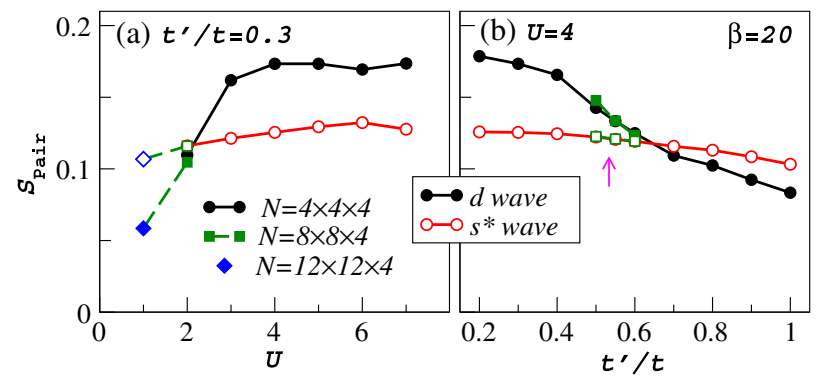

FIG. 4 (color online). (a) Pairing structure factor [29] at $\beta=20$ and $t^{\prime} / t=0.3$ vs the interaction strength. For $U=1$ and 2, two different system sizes are shown. (b) Pairing structure factor at $\beta=20$ and $U=4$ vs the ratio $t^{\prime} / t$. Full (empty) symbols are for the $d$-wave (extended $s$-wave) symmetry. The error bars are smaller than the symbols. The arrow indicates the location of the AF phase transition. 

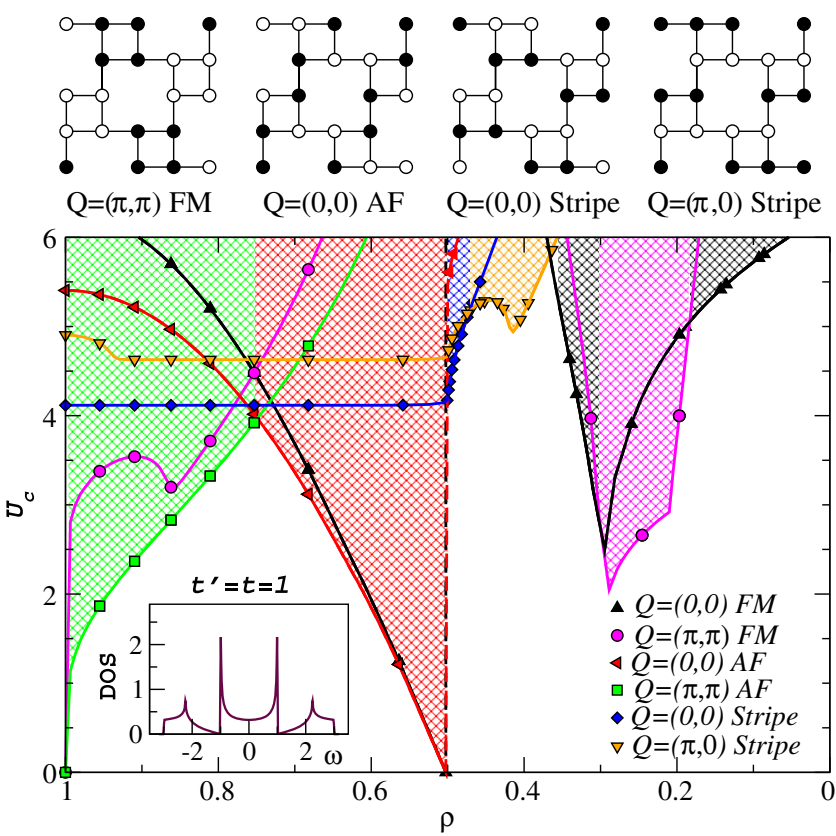

FIG. 5 (color online). (Top) Four of the magnetic orderings considered in this study. The up (down) spins are denoted by filled (empty) circles. $Q$ indicates the phase between unit cells and the FM, AF, and stripe denote the ordering of spins within each unit cell. (Bottom) The ground state RPA phase diagram of the model at $t^{\prime}=t$ away from half filling. The inset shows the density of states (DOS).

different doping regions. Figure 5 provides the full evolution of the critical interaction strength $U_{c}$ for six different magnetic orderings as a function of the electron density $\rho$ in the uniform $t=t^{\prime}$ case. Four of the magnetic phases are shown atop the main panel in Fig. 5. The other two are the regular $Q=(\pi, \pi) \mathrm{AF}$ (shown in Fig. 1) and the simple $Q=(0,0)$ ferromagnetic (FM) phases. Here, $Q$ is the wave vector corresponding to the superlattice and the following letters describe the order within a plaquette. The $Q=(\pi, \pi)$ AF dominates near half filling and up to $\rho \sim 0.75$. At that point, the $Q=(0,0) \mathrm{AF}$ has the largest susceptibility and hence, the smallest $U_{c}$, even though for densities close to, but higher than $\rho=0.5$ (quarter filling), it is degenerate with the $Q=(0,0) \mathrm{FM}$ phase. Exactly at quarter filling, we find that the $Q=(0,0)$ stripe, and not the $Q=(0,0) \mathrm{AF}$, as predicted by a previous mean-field calculation [37], is the dominant order. However, at a slightly smaller $\rho$, this order is replaced by the $Q=(\pi, 0)$ stripe order. Interestingly, at $\rho \sim 0.2$ to 0.3 , the block $\mathrm{AF}$ phase, observed in ordered-vacancy iron selenide materials, has the lowest $U_{c}$. This order shows up at even lower energies in the anisotropic case of $t^{\prime}<t$. Thus, in many ways, the single-orbital model at different dopings captures the richness of the magnetic phases observed in the iron pnictide and chalcogenide family of materials.

Quantum Monte Carlo methods allow for an exact treatment of the combined effects of correlation and band structure on lattices of finite spatial size, or equivalently, with finite resolution in momentum space. Previous DQMC studies of the effect of multiple bands and different intersite hoppings on magnetic order have mostly been confined to layered geometries in which two spatially extended regions each with a unique hopping are coupled [41-43]. Here, in contrast, we have presented results for a hopping pattern in which two different $t_{i j}$ are mixed locally, and found that tuning their ratio leads to multiple quantum phase transitions and rich phase diagrams. We have also studied the superconducting properties of our model at half filling within the DQMC method. Remarkably, the dominant pairing symmetry changes character from $d$ wave in the plaquette phase to an extended $s$ wave in the Néel phase, revealing an interesting interplay between magnetic and superconducting correlations. Although our system is insulating at half filling, the dominant pairing at half filling should be an indicator of the nature of superconductivity upon doping. Moreover, the behavior of both the magnetic and superconducting correlations in our single-orbital model offer surprising connections to iron-based superconductors, which are multiorbital systems, implying that they can be mapped to effective one-orbital models but with varying doping values.

This work was supported by the Department of Energy under Grant No. DE-NA0001842-0 (E. K. and R. T. S.) and by the National Science Foundation Grants No. PIF1005503 (R. T. S.), No. DMR-1004231 and No. DMR1306048 (E. K. and R. R.P. S.), and No. DMR-1207622 (W.E.P.). This work used the Extreme Science and Engineering Discovery Environment under Project No. TG-DMR130143, which is supported by NSF Grant No. ACI-1053575. We thank D. Chicks for additional useful input.

[1] E. Dagotto, Rev. Mod. Phys. 66, 763 (1994).

[2] G. R. Stewart, Rev. Mod. Phys. 83, 1589 (2011).

[3] M. J. Han, Q. Yin, W. E. Pickett, and S. Y. Savrasov, Phys. Rev. Lett. 102, 107003 (2009).

[4] I. I. Mazin and M. D. Johannes, Nat. Phys. 5, 141 (2009).

[5] P. Dai, J. Hu, and E. Dagotto, Nat. Phys. 8, 709 (2012).

[6] C. de la Cruz, Q. Huang, J. W. Lynn, J. Li, W. R. II, J. L. Zarestky, H. A. Mook, G. F. Chen, J. L. Luo, N. L. Wang et al., Nature (London) 453, 899 (2008).

[7] C. Xu, M. Müller, and S. Sachdev, Phys. Rev. B 78, 020501 (2008).

[8] A. V. Chubukov, D. V. Efremov, and I. Eremin, Phys. Rev. B 78, 134512 (2008).

[9] Q. Si and E. Abrahams, Phys. Rev. Lett. 101, 076401 (2008).

[10] G. S. Uhrig, M. Holt, J. Oitmaa, O. P. Sushkov, and R. R. P. Singh, Phys. Rev. B 79, 092416 (2009).

[11] I. I. Mazin, D. J. Singh, M. D. Johannes, and M. H. Du, Phys. Rev. Lett. 101, 057003 (2008). 
[12] R. M. Fernandes, D. K. Pratt, W. Tian, J. Zarestky, A. Kreyssig, S. Nandi, M. G. Kim, A. Thaler, N. Ni, P. C. Canfield et al., Phys. Rev. B 81, 140501 (2010).

[13] R. M. Fernandes and J. Schmalian, Phys. Rev. B 82, 014521 (2010).

[14] K. Okazaki, Y. Ota, Y. Kotani, W. Malaeb, Y. Ishida, T. Shimojima, T. Kiss, S. Watanabe, C.-T. Chen, K. Kihou et al., Science 337, 1314 (2012).

[15] Q. Q. Ge, Z. R. Ye, M. Xu, Y. Zhang, J. Jiang, B. P. Xie, Y. Song, C. L. Zhang, P. Dai, and D. L. Feng, Phys. Rev. X 3, 011020 (2013).

[16] N. Xu, P. Richard, X. Shi, A. van Roekeghem, T. Qian, E. Razzoli, E. Rienks, G.-F. Chen, E. Ieki, K. Nakayama et al., Phys. Rev. B 88, 220508 (2013).

[17] N. Katoh and M. Imada, J. Phys. Soc. Jpn. 64, 4105 (1995).

[18] K. Ueda, H. Kontani, M. Sigrist, and P. A. Lee, Phys. Rev. Lett. 76, 1932 (1996).

[19] M. Troyer, H. Kontani, and K. Ueda, Phys. Rev. Lett. 76, 3822 (1996).

[20] M. P. Gelfand, Z. Weihong, R. R. P. Singh, J. Oitmaa, and C. J. Hamer, Phys. Rev. Lett. 77, 2794 (1996).

[21] W. E. Pickett, Phys. Rev. Lett. 79, 1746 (1997).

[22] S. Taniguchi, T. Nishikawa, Y. Yasui, Y. Kobayashi, J. Takeda, S.-I. Shamoto, and M. Sato, J. Phys. Soc. Jpn. 64, 2758 (1995).

[23] B. Wei, H. Qing-Zhen, C. Gen-Fu, M. A. Green, W. DuMing, H. Jun-Bao, and Q. Yi-Ming, Chin. Phys. Lett. 28, 086104 (2011).

[24] F. Ye, S. Chi, W. Bao, X. F. Wang, J. J. Ying, X. H. Chen, H. D. Wang, C. H. Dong, and M. Fang, Phys. Rev. Lett. 107, 137003 (2011).

[25] E. Dagotto, Rev. Mod. Phys. 85, 849 (2013).

[26] R. Thomale, C. Platt, W. Hanke, J. Hu, and B. A. Bernevig, Phys. Rev. Lett. 107, 117001 (2011).

[27] S. Maiti, M. M. Korshunov, T. A. Maier, P. J. Hirschfeld, and A. V. Chubukov, Phys. Rev. Lett. 107, 147002 (2011).

[28] R. Blankenbecler, D. J. Scalapino, and R. L. Sugar, Phys. Rev. D 24, 2278 (1981).
[29] See Supplemental Material at http://link.aps.org/ supplemental/10.1103/PhysRevLett.113.106402, which includes Refs. [30-32], for a discussion of the DQMC method, finite-size scaling of the AF structure factors, and a brief discussion of the RPA.

[30] The DQMC codes under the QUantum Electron Simulation Toolbox (QUEST) project, which is used in this study, can be found at https://code.google.com/p/quest-qmc/.

[31] D. A. Huse, Phys. Rev. B 37, 2380 (1988).

[32] J. Kanamori, Prog. Theor. Phys. 30, 275 (1963); J. E. Hirsch and D. J. Scalapino, Phys. Rev. Lett. 56, 2732 (1986); N. E. Bickers, D. J. Scalapino, and S. R. White, Phys. Rev. Lett. 62, 961 (1989); A. V. Chubukov and D. M. Frenkel, Phys. Rev. B 46, 11884 (1992); N. Bulut, D. J. Scalapino, and S. R. White, Phys. Rev. B 47, 2742 (1993).

[33] E. Y. Loh, J. E. Gubernatis, R. T. Scalettar, S. R. White, D. J. Scalapino, and R. L. Sugar, Phys. Rev. B 41, 9301 (1990).

[34] W.-F. Tsai and S. A. Kivelson, Phys. Rev. B 73, 214510 (2006).

[35] Q. Luo, A. Nicholson, J. Riera, D.-X. Yao, A. Moreo, and E. Dagotto, Phys. Rev. B 84, 140506 (2011).

[36] H. Li and Y. Liu, Europhys. Lett. 98, 47006 (2012).

[37] Y. Yamashita, M. Tomura, Y. Yanagi, and K. Ueda, Phys. Rev. B 88, 195104 (2013).

[38] Y. Yanagi and K. Ueda, Phys. Rev. B 90, 085113 (2014).

[39] For $U=0$, exact results for clusters as large as $N=1024$ and at lower temperatures lead to $t^{\prime} / t=0.5$ as the location of the HSP.

[40] D. J. Scalapino and S. A. Trugman, Philos. Mag. B 74, 607 (1996).

[41] R. T. Scalettar, J. W. Cannon, D. J. Scalapino, and R. L. Sugar, Phys. Rev. B 50, 13419 (1994).

[42] A. Euverte, F. Hebert, S. Chiesa, R. T. Scalettar, and G. G. Batrouni, Phys. Rev. Lett. 108, 246401 (2012).

[43] A. Euverte, S. Chiesa, R. T. Scalettar, and G. G. Batrouni, Phys. Rev. B 88, 235123 (2013). 